\title{
A Framework for Fully Automated Home using loT Reliable Protocol Stack and Smart Gateway
}

\author{
P.C. Prabhu Kumar ${ }^{*}$, P. Penchala Prasanth, P. Hemalatha and Karthik J Kulakarni
}

Dept of CSE Audisankar College of Engineering Nellor, India

\begin{abstract}
A fully automated house must ensure all the appliances must be connection and provide a smart way of working for the human. The insight of Internet of Things (IOT) network provision the necessary platform to implement the automated home. The proposed system emphasizes, how all the appliances would be connected to loT to ensure fully automated home. In this framework the smart home has been divided into various areas like smart kitchen, smart gardening, home safety and security system, and smart lightning system. The reliable protocol stack has been utilized to provide efficient communication along with proper security measures. The reliable protocol suite works on top of MQTT and TCP to ensure reliable communication. The smart gateway utilized for this framework and provides firewall security as with a two-phase filtering mechanism as well as scalability. among all the appliances in the home.
\end{abstract}

Keywords: Internet of things (loT), Smart home, MQTT, Raspberry Pi, Gateway.

\section{INTRODUCTION}

The association of loT in home automation facilitates a smart approach and brilliant features to satisfy the daily needs of the human beings. IoT is an umbrella technology, which contains sensors, wireless communication, cloud computing, bigdata, and so on. These technologies makes loT more flexible pertaining constrained environment. IoT applications like home automation involves a greater number of sensors for collecting the data and above-mentioned technologies provisions sophisticated way of data collection, networking, storing, computations, security measures and analyzing. Home automation provides monitoring the conditions of home remotely and actuating the respective action. The proposing system emphasizes various types of home applications similar to smart lighting, smart bathroom, smart gardening, home safety and security mechanism, smart kitchen, measuring air and water quality, voice assistant for elderly people, smart infotainment devices, smart switches, smart locks, smart energy meters, and Al based digital experiences.

The basic operation in automated smart home is the sensors would collect the data and that data will be transmitted to the loT gateway embedded with raspberry $\mathrm{pi}$, finally the data will be stored and analyzed in cloud to perform appropriate actuation process. Users can monitor entire process through exclusive mobile applications. In the market various loT platform are available, which provides platform as a

*Address correspondence to this author at the Dept of CSE Audisankar College of Engineering Nellor, India; E-mail: drprabhu.cse@audisankara.ac.in; prasanthsvcn@gmail.com; hema.keshav10@gmail.com service such as IBM Bluemix, Azure loT, Thingworx, Carriots, Ubidots, Konekt, Thingspeak, TempolQ, Xively, and AWS IoT. These providers facilitate the platform services for analytics, data visualization, storage, remote monitoring, and controlling.

The organization structure of the paper is as follows: in section II, a brief overview pertaining reliable protocol stack and smart gateway has been presented. Section III is about the related work that was already done in smart home domain. In section IV the prototype of the proposed system is explained. Section V describes the conclusions and future scope of the proposed work.

\section{RELATED WORK}

The authors [7] explained about the architectures for smart home automation and proposed a novel home automation architecture using all the loT protocols. The authors [8], proposed a prototype to perform home automation using SMS. They used GSM network and the micro-controller devices. This proposed system also emphasizes on the security issues in the networking and proposed a secure, reliable and adaptable smart home system. The research work done in [9] provides the comparison between MQTT and HTTP and concluded that MQTT is better than HTTP for the constrained nodes with limited resources. This research also proved that, the data transmission using MQTT consumes only about $0.05 \%$ of battery/hour by using 3G for network connectivity. The authors [10] designed a protocol suite especially for loT applications which contains a greater number of sensors and loT node, they emphasized on reliable communication between the sensor nodes, loT device 
and gateway. This paper explained a prototype overview of a light weight Message Queuing Telemetry Transport (MQTT) protocol. The authors focused on implementation of MQTT on ESP8266 (Node MCU) embedded with a WiFi based development board and sensors and actuators would be connected to ESP8266 and a Mosquitto [11] [12] based MQTT broker is used for remote monitoring and control.

\section{RELIABLE PROTOCOL STACK AND SMART GATEWAY}

\subsection{Reliable Protocol Stack}

The Reliable protocol stack ensures reliable and secure interaction among the home appliances, IoT devices, and smart gateway. This stack works with TCP and TSL/SSL protocols, hence it has been named as reliable. Table 1 defines the respective protocols at each layer to ensure reliable communication.

Table 1: Reliable Protocol Stack

\begin{tabular}{|c|c|}
\hline Layer Name & Feasible Protocols \\
\hline \hline Application Layer & MQTT \\
\hline Transport Layer & TCP, TLS, SSL \\
\hline Network Layer & IPv6, 6LoWPAN \\
\hline Link Layer & Bluetooth, WiFi \\
\hline
\end{tabular}

This reliable suite provides lightweight machine to machine communication, and this works on top of MQTT application protocol. MQTT ensures reliable publish and subscribe communication, which utilizes the network bandwidth with a 2-byte header [1]. In MQTT, each sensor node acts as a client and establishes a connection to a broker. Every message in MQTT is a different collection of data and the data message is known as topics. In MQTT the publishers, publish the data message on the brokers, and subscribers receive the respective data. First the MQTT client sends a request to MQTT broker to set a connection. Then broker performs validation and returns the connection acknowledgement. Once the connection has been established then the client publishes the data and it would be ensured by broker with a publish acknowledgement. If the communication is done then the client sends a disconnect message to the broker. Figure 1 defines the structure of the MQTT protocol with publishers, subscribers, and the brokers. Figure 2 shows the interaction between MQTT client and MQTT broker. CoAP is another important application protocol for loT applications, but it is not sutable for smart home application since it supports one to one communication [2]

As shown is Table 2, the reliable protocol stack supports The Transmission Control Protocol (TCP) at transport Layer, since the virtue of MQTT is reliable as well as connection oriented and it will be achieved by TCP. The Transport Layer Security (TLS) and Secure Socket Layer (SSL) ensure necessary security measures.

TLS provides the authentication security among publishers, subscribers, and brokers through the usernames and passwords and SSL ensures a secure channel between the clients and brokers.

In network layer the reliable protocol stack supports IPv6 and 6LoWPAN protocols are proposed, since to address the billions of loT devices through the internet. The IPv6 is a protocol for packet-switched internetworking and provides end to end datagram communication for IP networks. 6LoWPAN is an alternate of IPv6 pertaining Low power Wireless Personal Area Networks with IPv6 addressing. The compatible protocols of reliable stack for link layer are

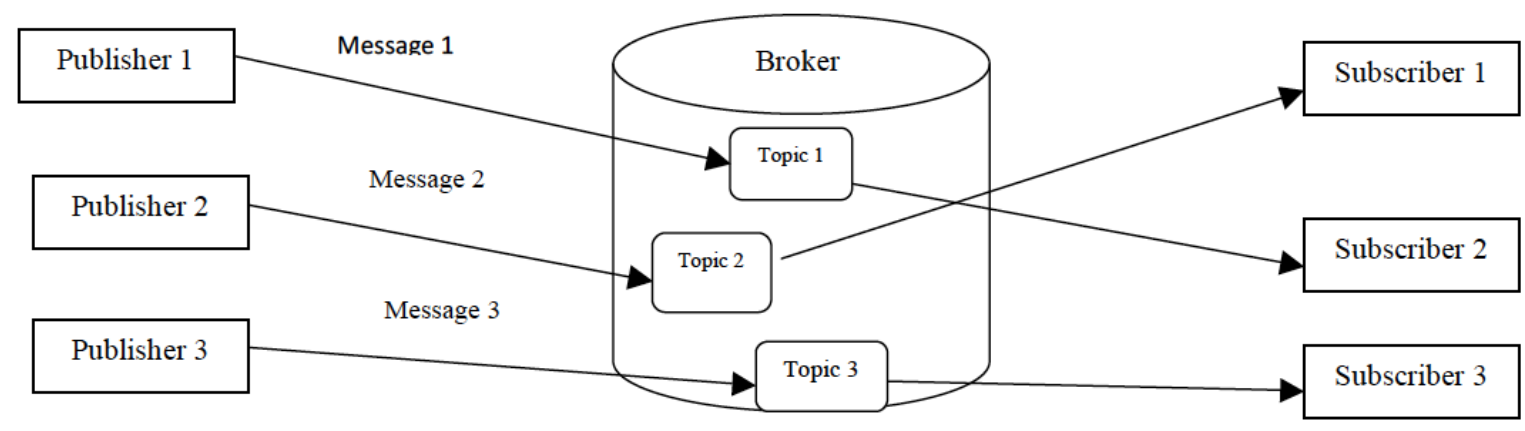

Figure1: The structure of MQTT protocol. 
Bluetooth and WiFi. Bluetooth is a wireless communication protocol for shorter distance between the range $2.4 \mathrm{GHz}$ to $2.45 \mathrm{Ghz}$ ISM band and the data rate is $3 \mathrm{Mbps}$ for maximum distance $100 \mathrm{~m}$. For WiFi, the frequency ranges are $2.4 \mathrm{GHz}, 3.6 \mathrm{GHz}, 4.9 \mathrm{GHz}$, and $5.9 \mathrm{GHz}$.



Figure 2: MQTT Client and Broker interaction.

The maximum data rate ranges from $54 \mathrm{Mbps}$ to $600 \mathrm{Mbps}$ for $\mathrm{WiFi}$. The protocol discussed so for constructs sophisticated networks among the home appliances, IoT nodes, and the smart gateway, the implementation would be defined in further sections.

\subsection{Smart loT Gateway}

The insight capabilities of the loT node are limited concerning process, power, storage, and computation. Hence, the constrains of the loT node are addressed through the sophisticated and robust middleware. The smart gateway reduces the overhead of the constrained devices of loT. The smart loT gateway is an important aspect of constructing a secure, efficient, easy-to-maintain, and self-configurable system [3]. The IoT smart gateways integrate various heterogeneous networks and also work as mediators between constrained sensing networks and mobile networks. The objective of loT gateway is, it collects the data from sensors of WSNs and forwards the data to local computation system or cloud computing through the internet, and it also collects the information from the cloud, which is then forward to the device to perform respective actuation function, such as triggering environmental changes and identifying possible issues with functioning [4]. The following limitations demystify the importance of smart gateway for constrained loT environment. The first issue is, the sensors usually have limited capabilities concerning networking connectivity. Another important challenge with respect to range networking protocols for WSN (Wireless Sensor Network), such as BLE (Bluetooth Low Energy), Zigbee, and IEEE 802.15.4 cannot be connected directly to more extensive networks similar to Wide Area Network (WAN) through the internet. A gateway would play important role to connect the sensors with external heterogeneous networks by using Wi-Fi, GSM, or other types of connectivity. The second limitation with the loT end nodes applications is that the processing and computation capabilities are limited. That is, these nodes would have low configured processors and internal memory and it is difficult to perform the complex operations or analytics. The smart loT gateway would address these constrains by performing all the computations and ensures less overhead at end nodes [5]. Another issue with sensor nodes is security and they are vulnerable to attack. The data generated by these nodes are also at risk. The end nodes of loT network would not have sufficient security measures, since the computation capabilities of these devices limited. Hence the smart gateway ensures the required security for the end device as well as the data generated, by employing authentication and authorization and various encryption techniques.

The gateway was designed through the raspberry$\mathrm{pi}$, hence it would get the additional features such as, a smart way of converting the protocols between native internet and constrained network, provides a secure interface with efficient algorithms for constrained loT environment. The gateway contains a centralized proxy mechanism, which ensures a flexible way of translating the protocols among various heterogeneous networks [6]. The gateway also contains a two-way filtering mechanism to establishing the secure channel as well as filtering the packets. The one more feature of the gateway is flexibility, the raspberry pi and switch controllers provides the number of ports to connect multiple number of sensors and other devices. The smart gateway with the association of reliable protocol stack gives a robust middleware structure for proposed smart home framework. Figure $\mathbf{3}$ shows the important components and structure of smart and secure loT gateway [10]. 


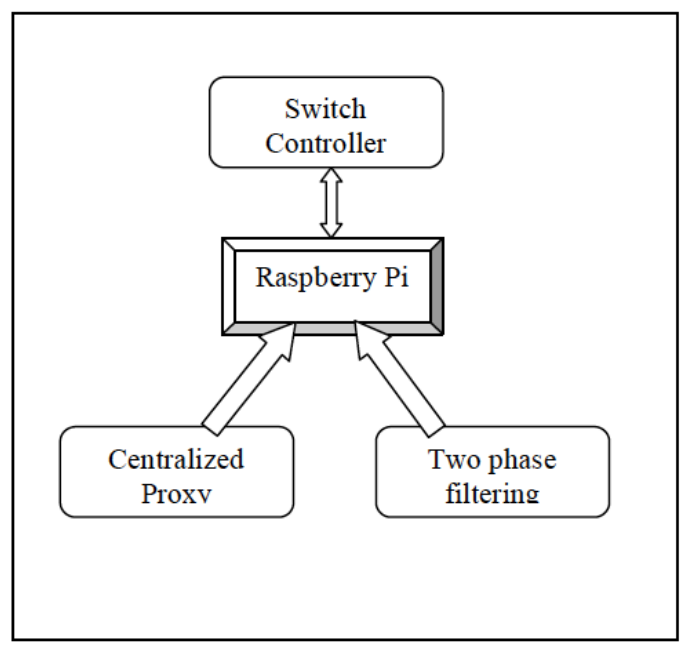

Figure 3: Components of smart gateway.

\section{FRAMEWORK FOR AUTOMATED HOME}

In the proposed system the automated smart home frame work has been segregated into area and room wise. The following section explains the detailed way of transforming the home appliances into the smart loT things to provide automated way of communication among them to serve humans. The total home automation has been divided into five areas, like

\section{- $\quad$ Smart Kitchen}

- $\quad$ Smart Gardening

- $\quad$ Home safety and security system

- $\quad$ Smart Lightening system

- $\quad$ Al driven voice based assistance

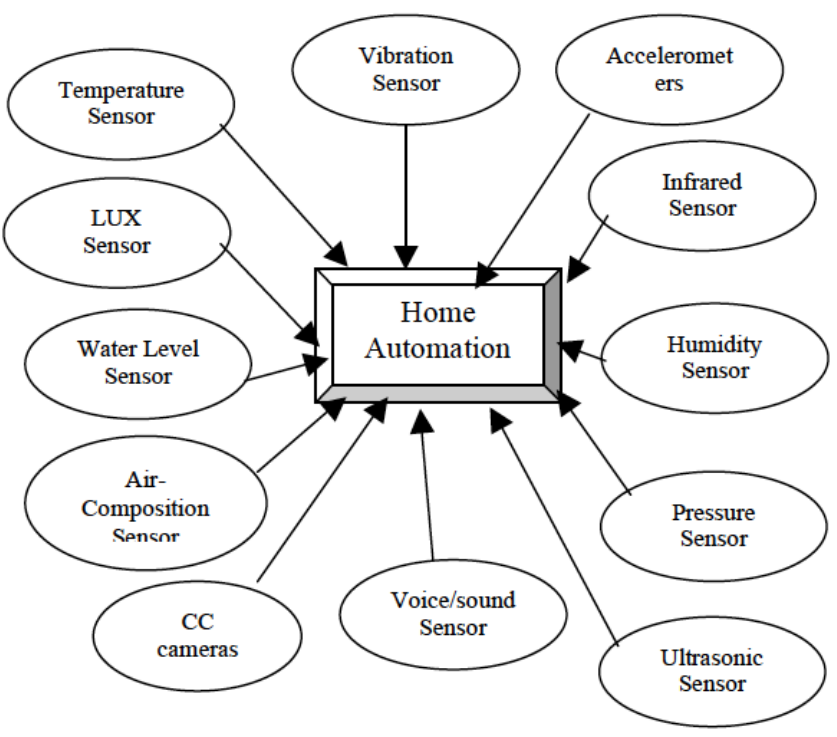

Figure 4: Sensors for home automation.
Sensors plays an important role in home automation for collecting the information from environment and respective home appliances. Sensor collects the raw data and forwards to loT device for analytics. Figure 4 defines various sensors used in the application of home automation. The temperature sensor collects the current temperature of the room. The important sensors for temperature are MSP430 DHT11/22, LM35 and DS18B20. Among these the MSP430 sensors consumes less power and battery. The ultrasonic sensor is used to measure the water level and this sensor is used to calculate the levels of water in water tanks. One variant of water level ultrasonic senor is HC-SR04 ultrasonic sensor. The air composition senor is used to measure the different compositions of the air such as $\mathrm{CO}$, Hydrogen gas, Nitrogen Oxide, and other hazardous gases. There are multiple air sensors are present to calculate the composition of certain gases such as MICS-5525 for CO, MQ-8 for Hydrogen, MICS2714 for Nitrogen, and MQ135 for other gases. The sensors would give the accurate values, when their surface has been heated enough. The LUX sensor is used to measure the luminosity and it is mainly used in the room for smart lighting system. The best LUX sensors are TSL2591 and BH1750. The surveillance cameras are used to capture the videos. The gateway used in this framework is manufactured using Raspberry $\mathrm{Pi}$ and its provisions a built-in camera module, hence no need to use USB port to connect the cameras. Sound sensors are used to measure the units of sound and it is easy to monitors the smart lightning system with respect to the sounds of babies in the home. Another objective of the sound senor is to design the voice based automated system to monitor various appliances in the home. The humidity sensors are used to measure the $\mathrm{RH}$ (Relative Humidity) levels in the air, and these sensors can be used for smart cooling system. The variants of humidity sensors are HIH6100, Dig RH, DHT22, and DHT11. Accelerometers are used to detect vibration and accelerations. This sensor would be mainly used in cellphones. Infrared sensor emits the light to sense objects and used for security system to detect the unauthorized objects.

\subsection{Smart Kitchen}

The loT devices and related sensors turns the normal kitchen into a smart kitchen to ensure great efficiency in the food preparation and cooking processes. Smart kitchen associates the smart appliances like refrigerator, coffeemaker, oven, stoves, smoke emitters, and dishwasher. This section explains 
the smart working of each appliance with respective sensors and loT devices.

- Refrigerator plays a vital role in kitchen to preserve the vegetable and various food items. A smart refrigerator provisions more features such as monitoring the lifetime of the vegetables, expiry date of the food products, number of eggs in the tray and the status. The refrigerator also sends the alerts to the user as well as storekeeper about the requirement of the food products which are in less quantity. Based on the season and the room temperature it adjusts the cooling inside the fridge.

- Coffee is an important source of healthful antioxidants and most of the people are addicted to it. Hence, the coffee maker is an essential kitchen appliance. Coffeemaker can be wirelessly connected to the phone through Bluetooth or Wi-Fi and it can be operated using a mobile application interface. Hence based on the coffee requirement and the ingredients instantly it can be prepared by smart coffee maker.

- A smart oven is a kitchen appliance that is similar to a normal microwave but contains extra features like remember and managing the cooking times, cook food evenly, and prevent items from burning. A smart oven would be equipped with a scanner, which can scan the bar codes of various food products. Hence the smart scanner can then program the oven to the feasible temperature and set the required amount of time for the item to cook. This can provisions proper cooking and ensure no burnings. The smart oven also contains the technology to generate the heat from all sides and ensures the less consumption of electric power. A mobile application will be used to operate the oven remotely through the mobile phone.

- $\quad$ Minimizing time and energy while preparing food using smart stoves has become an important part of human lives. A smart stove helps to prepare a well-cooked food on time and can be controlled from remote by using certain mobile application. This remote control technology through internet has allowed to be efficient and flexible. Hence the users can spend the time to do other works.
- A smart dishwasher is an important kitchen appliance to clean all the kitchen vessels. A smart dish washer is a connected smart home appliance and provides enhanced features like Wi-Fi connectivity and flexible options such as voice controlling through the enabled virtual home assistant and mobile phone interface. This washer facilitates the start wash cycles anytime, anywhere from a smartphone to monitor performance and check cycle status. The smart dishwasher also sends the alerts through mobile app enabled smartphone like rinse aid or detergent is low, when a wash cycle completes, or if the sensors detect problem with hardware or leak.

\subsection{Smart Gardening}

A beautiful lawn increases the beauty of the house, to design a smart garden the following areas must be focused.

- Smart plant monitors or sensors: There are various plant monitor sensors are available for smart gardening. These sensors are simply inserted into soil and takes the measurements of moisture levels inside the soil, track light and humidity values, and even analyze soil properties. Some sensors can even determine the nutrients in soil.

- $\quad$ Smart water sprinklers: Smart water sprinklers do more than just schedule irrigation. They also identify any breaks or leaks in the watering system, save water, adjust to respective weather and often can be controlled and changed through the mobile phone or computer system.

- Expandable container pots: It is a wonderful technology regarding expandable pot. The containers would expand as the plant grows, so no need to keep purchasing pots a size up when plants grow.

- Smart Gardening applications: Garden applications can help with design, plant ID, placement of irrigation, solve problem areas and much more. Many, like the GKH Gardening Companion (for Android and iPhone), are available for free of cost or purchase easy-to-use guides in a variety of formats.

- $\quad$ Smart mowers - Mowbot is a smart lawn mower. It works same as to robotic vacuums and it ensures no more sweating in the hot by humans. 


\subsection{Home Safety and Security System}

Most of the home safety measures deals about the importance of preventing fire accidents, safety against extreme weather conditions and protecting the home from potential burglars. There are various smart ways to protect the home from common risks and dangers.

- Detecting odourless and colourless gas mainly $\mathrm{CO}$, that is carbon monoxide detector is one of the most important aspect, $\mathrm{CO}$ originates from many sources, such as gas and diesel sources, like vehicles and generators, Fireplaces, Space heaters, Wood stoves, and Oil, propane or natural gas appliances. The $\mathrm{CO}$ detector measures the amount of Carbon monoxide in the air, it is good to install the CO detector 5 feet away from the kitchen appliances.

- Home security alarms are mandatory home safety mechanism to get the alerts about potential intruders, burglars, thieves, and other emergencies.

- $\quad$ Other important safety measures, which should be addressed are locking the medicine cabinets, Keeping the poisonous and dangerous chemicals out of reach. Covering sharp corners with foam or soft padding, taking care of gates near staircases for child concern. The respective sensors would ensure the proper measures with respect to above concerns.

\subsection{Smart Lighting System}

The objective of smart lighting is to turns off/on themselves without any human intervention based on the sensor values. The best light sensors are LUX TSL2591 and BH1750. The smart lighting system, not only save the time but will also help to save the electricity that is a non-renewable resource. The intensity of the bulb would also be controlled with the help of smartphones. The sensors, which would detect the objects sends the signals to the actuators to ON the lights based on the signals generated by the loT devices. Figure 5 gives the structure of smart home, where the home appliances, sensors and the actuators are connected to smart gateway, the analytics can be performed either in the local system or in the cloud, based on the volume of sensor data [13]. The smart gateway would be augmented with reliable protocol suite as well as two-way filtering mechanism to ensure the necessary security [14].

\section{CONCLUSION AND FUTER WORK}

In the proposed framework the fully automated home is implemented with a reliable protocol suite and smart gateway. The reliable protocol suite ensures many to many reliable communications among various sensors and home appliances. The smart gateway provides required security measures as well as flexible way of protocol translation among constrained IoT environment, native internet, and cloud computing. The proposed framework also explained various modules such as smart kitchen, smart gardening, smart lighting system, and home security system [15]. These modules give enough information to convert the normal home into a fully automated home including the respective sensor and the actuator. Taking this further ahead, implementation of proposed framework would be done. In further study the frameworks will also be developed and implemented using suitable protocol

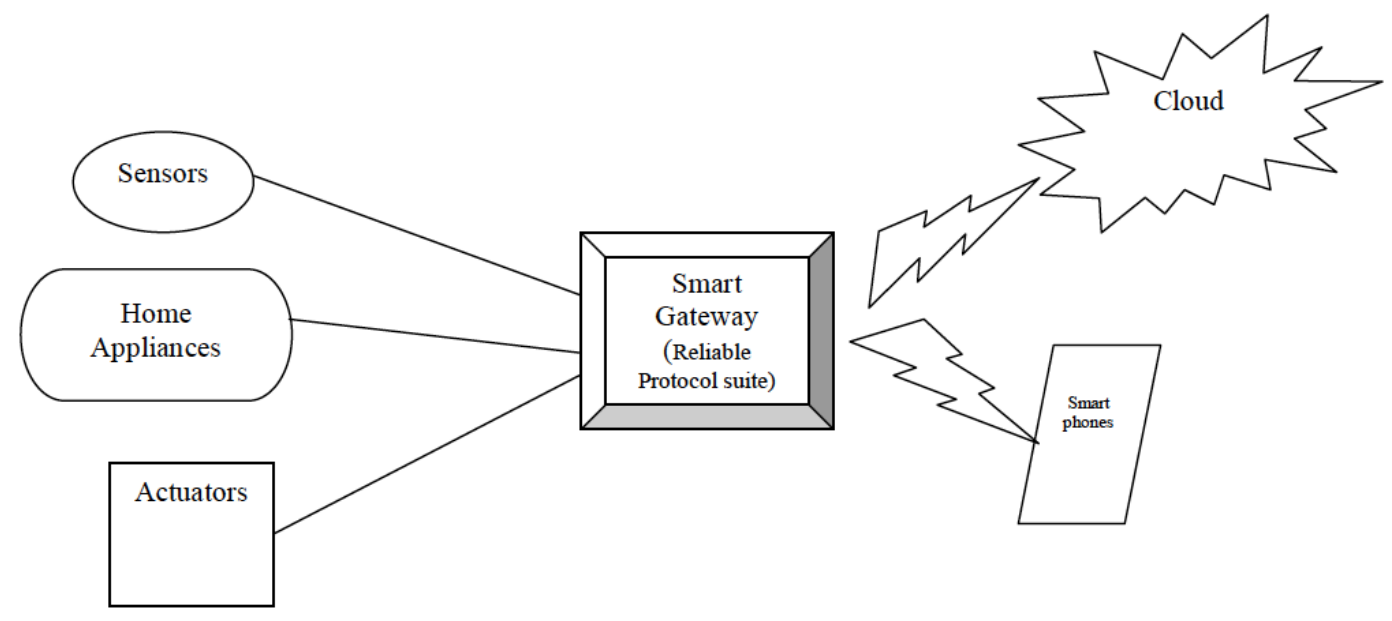

Figure 5: Structure of smart home. 
suite and smart gateway for various loT applications such as smart city, IloT (Industry loT), Smart logistics, Smart Irrigation, Smart Health system, etc.

\section{REFERENCES}

[1] Mqtt v3.1 protocol specification. [Online]. Available: http://public.dheibm.com/software/dw/webservices/wsmqtt/mqtt-v3r1.html

[2] Christine Hennebert and Jessye Dos Santos, Security protocol in 6LoWPAN Stack: A Synthesis, IEEE Internet of Things Journal 2016; 1(5): 384-398. https://doi.org/10.1109/JIOT.2014.2359538

[3] Byungseok Kang, Daecheon Kim, and Hyunseung Choo. Internet of Everything: A Large-, IEEE Transactions On MultiScale Computing Systems, 2017; 3(3): 206-215. https://doi.org/10.1109/TMSCS.2017.2705683

[4] Christine Hennebert and Jessye Dos Santos, Security protocol in 6LoWPAN Stack: A Synthesis, IEEE Internet of Things Journal 2016; 1(5): 384-398. https://doi.org/10.1109/JIOT.2014.2359538

[5] Guoqiang, S, Yanming, 2013, Design and implementation of a smart loT gateway in Green Computing and Communications (GreenCom), IEEE and Internet of Things (iThings/CPSCom), IEEE International Conference on and IEEE Cyber, Physical and Social Computing, pp. 720-723. https://doi.org/10.1109/GreenCom-iThingsCPSCom.2013.130

[6] Chen, Xueqin Jia, Heng Li, A brief introduction to loT gateway, Communication Technology and Application (ICCTA 2011), IET International Conference on, 2011; pp. 610-613.
[7] S. Nasrin and PJ. Radcliffe, "Novel protocol enables diy home automation," in Telecommunication Networks and Applications Conference (ATNAC), 2014 Australasian, 2014; pp. 212-216.

https://doi.org/10.1109/ATNAC.2014.7020900

[8] H. ElKamchouchi and A. ElShafee, "Design and prototype implementation of sms based home automation system," in Electronics Design, Systems and Applications (ICEDSA), 2012 IEEE International Conference on, 2012; pp. 162-167. https://doi.org/10.1109/ICEDSA.2012.6507788

[9] A. Kumar and S. Johari, "Push notification as a business enhancement technique for e-commerce," in 2015 Third International Conference on Image Information Processing (ICIIP), Dec 2015, pp. 450-454. https://doi.org/10.1109/ICIIP.2015.7414815

[10] Prabhu Kumar P C\& Geetha G, P levels and optimized MQTT and CoAP protocol suites in web of Wiley publication, 2019; 31(12).

https://doi.org/10.1002/cpe.4867

[11] Light, R 2013, Mosquitto-an open source mqtt v3.1 broker, http://mosquitto.org/.

[12] Eclipse. Mosquitto an open source mqtt v3.1/v3.1.1 broker [Online]. Available: http://mosquitto.org/

[13] Sye Loong Keoh, Sandeep, S. Kumar, Hannes 2016 Securing the IOT: IEEE Internet of Things Journal, 2016 1(3): pp. 265-275.

[14] Telecommunication Standardization Sector of ITU, Common requirements and capabilities of a gateway for Internet of things.

[15] Adam Zielonka, Marcin Woźniak, Sahil Garg, Smart Homes: How Much Will They Support Us? A Research on Recent Trends and Advances, IEEE Acess 2021; 9: pp 26388-26419 https://doi.org/10.1109/ACCESS.2021.3054575

DOI: http://dx.doi.org/10.31875/2409-9694.2020.07.7

(C) 2020 Kumar et al.; Licensee Zeal Press.

This is an open access article licensed under the terms of the Creative Commons Attribution Non-Commercial License (http://creativecommons.org/licenses/by-nc/3.0/), which permits unrestricted, non-commercial use, distribution and reproduction in any medium provided the work is properly cited. 